\title{
Modelo estructural del Campo Escuela Colorado a partir de modelos análogos
}

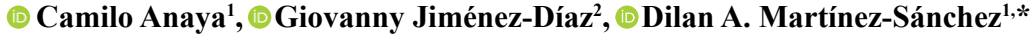 \\ ${ }^{1}$ Escuela de Geología, Universidad Industrial de Santander, Bucaramanga, Colombia \\ ${ }^{2}$ Grupo de Investigación en Geología Básica y Aplicada (GIGBA), Escuela de Geología, \\ Universidad Industrial de Santander, Bucaramanga, Colombia
}

\begin{abstract}
Resumen
La cuenca del Valle Medio del Magdalena es una cuenca intermontana (Cooper, et al., 1995; Taboada, et al., 2000; Sarmiento-Rojas, et al., 2006), que se caracteriza por tener estructuras con doble vergencia y está limitada por las cordilleras Central y Oriental (Córdoba, et al., 2000). En el sector sureste del Valle, asociado al frente de deformación occidental de la Cordillera Oriental, se localiza el Campo Escuela Colorado de la Universidad Industrial de Santander. Los estudios previos (Ecopetrol, 2003; Chajín, et al., 2013; Zafra, 2013) en dicho campo difieren en cuanto al modelo estructural y su relación con las estructuras regionales (por ejemplo, la falla de La Salina). En este trabajo se presentan los resultados de 15 modelos de simulación de una cuña crítica para definir las características estructurales en un ambiente compresivo. Los resultados de los modelos muestran un frente de deformación dominado por fallas con vergencia hacia el promontorio y retrocabalgamientos asociados. El apilamiento de material causó elevación topográfica en la zona axial de la cuña. Con base en la tendencia observada en los 15 experimentos realizados, se concluyó que la geometría de las estructuras está dominada por la escamación delgada y que el modelo estructural que define al Campo Escuela Colorado es una zona triangular controlada por un retrocabalgamiento. (C) 2018. Acad. Colomb. Cienc. Ex. Fis. Nat.
\end{abstract}

Palabras clave: Estilo estructural; Valle Medio del Magdalena; Campo Escuela Colorado; Modelos análogos.

Structural model of Campo Escuela Colorado based on analog models

\begin{abstract}
The Middle Magdalena Valley is an intermontane basin (Cooper, et al., 1995; Taboada, et al., 2000; SarmientoRojas, et al., 2006) characterized by double vergence structures and flanked by the Central and Eastern Cordilleras (Córdoba, et al., 2000). The Campo Escuela Colorado oil field of the Universidad Industrial de Santander is located in the South Eastern zone of the Valley, associated with the western deformation front of the Eastern Cordillera. Previous works on this oil field have diverged as regards the structural model and the relationship with major structures (e.g., La Salina Fault). We present the results of 15 critical wedge simulation models to define the main structural characteristics in a compressive tectonic setting. Our results showed a deformation front dominated by thrust faults that verge towards the foreland and by associated backthrusts. The topographic elevation is caused by material stacked in the axial zone of the wedge. Based on the 15 experimental models we concluded that the geometry of the structure is dominated by thin flaking and that the structural model of the Campo Escuela Colorado is a triangular zone dominated by a backthrust. (C) 2018. Acad. Colomb. Cienc. Ex. Fis. Nat.
\end{abstract}

Key words: Structural style; Middle Magdalena Valley; Campo Escuela Colorado; Analog models.

\section{Introducción}

El Valle Medio del Magdalena es una cuenca intermontana afectada por eventos de extensión, compresión, transcurrencia e inversión tectónica (Cooper, et al., 1995; Taboada, et al., 2000; Sarmiento-Rojas, et al., 2006). En estudios previos en el sector central-sur de la cuenca se evidenció un estilo estructural predominante con vergencia hacia el oeste (Mojíca \& Franco, 1990; Caballero, et al., 2010; López \& Jaimes, 2015) y presencia de zonas transversales (Restrepo-Pace, et al., 2004; Jiménez, et al., 2016). El Valle del Magdalena Medio se caracteriza por una deformación concentrada en la secuencia sedimentaria que no involucra basamento (Restrepo-Pace, et al., 2004; Jiménez, et al. 2016; López \& Jaimes, 2015), y se define como de escamación delgada (Cristallini, 1998). En márgenes convergentes,

\footnotetext{
*Correspondencia:

Dilan A. Martínez-Sánchez; dilanmsanchez30@gmail.com

Recibido: 18 de junio de 2018

Aceptado: 2 de octubre de 2018

Editor: Carlos Vargas Jiménez
} 
el acortamiento horizontal y el espesor de la secuencia sedimentaria son los dos aspectos principales que controlan la geometría de las cuñas de deformación, las cuales generan un ángulo con respecto a la horizontal (ángulo crítico $=\delta+$ $\varphi)$ (Davis, et al., 1983; Portillo, 2008). Uno de los métodos para el estudio de procesos relacionados con las cuñas de deformación es el análisis por modelamiento análogo. Este método se basa en el uso de materiales granulares como la arena. Los modelos análogos han demostrado una relación con la evolución estructural de las cuñas de deformación y las propiedades de los materiales utilizados, por lo tanto, el modelamiento es un acercamiento de primer orden para entender los mecanismos de los diferentes estilos de deformación presentes en la naturaleza (Malavieille, 1984).

Las cuñas acrecionales, cuñas de deformación o cinturones plegados y con fallas, así como las cuñas de arena en modelos análogos, se describen en términos del coeficiente de fricción interna de los materiales (Tang $\phi$ ) y el ángulo del despegue basal $(\mu \mathrm{b})$, los cuales controlan el ángulo de la topografía de la cuña $(\delta)$ y el ángulo de des-pegue basal $(\varphi)$ (Davis, et al., 1983; Portillo, 2008).
El análisis e interpretación de los datos mejoran considerablemente con el empleo de modelos conceptuales, mecánicos o cinemáticos basados en técnicas numéricas o experimentales (Graveleau, et al., 2012). Los modelos experimentales son útiles porque permiten observar geometrías y características distintivas de los procesos geológicos a escala de laboratorio. Estos modelos, además, son útiles y viables, pues pueden escalarse razonablemente (Brun, 2002; Hubbert, 1937).

Teniendo en cuenta que para el Campo Escuela Colorado, localizado en la cuenca del Valle del Medio del Magdalena (Figura 1), se han propuesto por lo menos tres modelos estructurales o hipótesis diferentes (Ecopetrol, 2003; Chajín, et al., 2013; Zafra, 2013), el propósito principal de este trabajo fue evaluar experimentalmente el estilo estructural de su sector sureste, con el fin de determinar cuál de dichos modelos era el más coherente.

\section{Marco geológico del Valle Medio del Magdalena}

Geología estructural. El Valle Medio del Magdalena es una depresión tectónica asimétrica, limitada por dos frentes

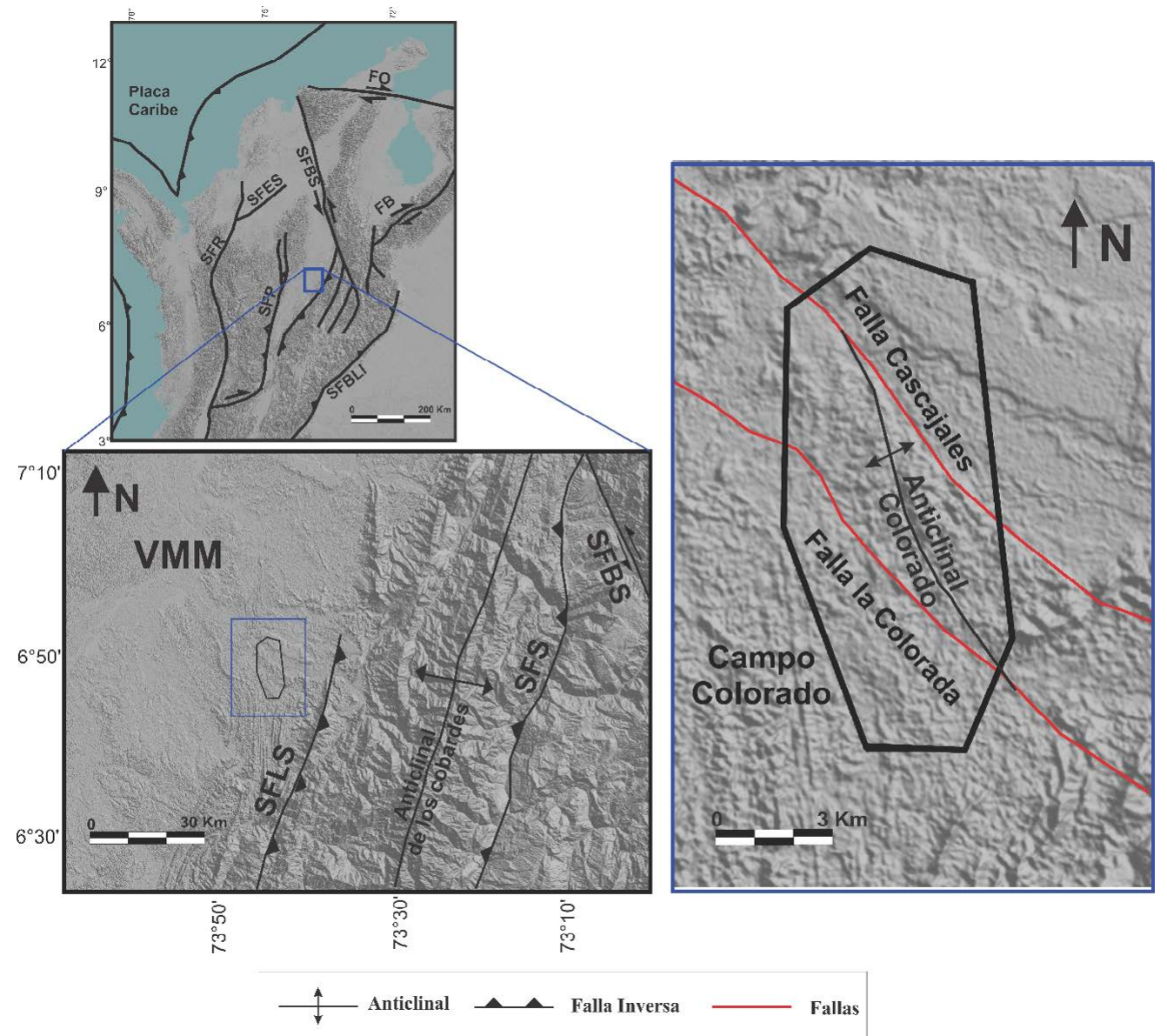

Figura 1. Modelo de elevación digital (DEM) con la ubicación del Campo Escuela Colorado en el contexto colombiano. VMM: cuenca del Valle Medio del Magdalena; FO: Falla de Oca. FB: Falla de Boconó; SFP: sistema de fallas de Palestina; SFR: sistema de fallas de Romeral; SFBLl: sistema de fallas del Borde Llanero; SFLS: sistema de fallas de Las Salinas; SFS: sistema de fallas de Suárez; SFBS: sistema de fallas de Bucaramanga- Santa Marta 
cabalgantes de vergencia opuesta, (Córdoba, et al., 2000). Está limitado al norte por el sistema de fallas de Espíritu Santo (S.F.E.S), al noreste, por el sistema de fallas de Bucaramanga-Santa Marta (S.F.B.S.M); al sureste, por el sistema de fallas de Bituima y La Salina (S.F.B.S), y al oeste, por la Serranía de San Lucas y la cordillera Central (Barrero, et al., 2007).

El Valle presenta diversos estados de deformación que se iniciaron con un periodo extensional durante el Jurásico y hasta el Cretácico temprano. Posteriormente, se produjo un periodo de subsidencia y flexura de la cuenca hasta el Eoceno, cuando las fases iniciales de la orogenia andina cambiaron el estado de la cuenca y la sometieron a procesos compresivos que resultaron en la generación de fallas inversas y la inversión de estructuras preexistentes (Sarmiento-Rojas, et al., 2006; Bayona, et al., 2008; Horton, et al., 2010; Moreno, et al., 2011; Nie, et al., 2012).

El Valle se ha definido estructuralmente en tres sectores (Córdoba, et al., 2001). El sector oriental, caracterizado por estructuras con dirección noreste-suroeste, corresponde predominantemente a sistemas de cabalgamientos con vergencia hacia el oeste. El sector central se encuentra poco deformado y allí se encuentran fallas normales fosilizadas. El sector occidental se caracteriza por la presencia de fallas inversas con componente transpresivo y vergencia hacia el este.

La deformación del Valle se incrementa hacia el sureste en un frente de deformación netamente compresional (Taborda, 1965). Su límite oeste se caracteriza por una baja deformación y por truncaciones de las formaciones cretácicas y acuñamientos de rocas cenozoicas (Mojica \& Franco, 1990).
Estratigrafía. La secuencia sedimentaria del Valle Medio del Magdalena está conformada por rocas cuya edad corresponde al Jurásico-Cretácico inferior (Berriasiano), asociadas a sistemas fluviales, con un control estructural (formaciones de Girón, Arcabuco-Los Santos) (EtayoSerna, 1968; Fabre, 1983), así como por rocas del Cretácico temprano depositadas en una plataforma marina somera (formaciones de Cumbre, Rosablanca, Paja y Tablazo), rocas con edad entre el Albiano y el Maastrichtiano que corresponden a ambientes marinos someros (formaciones de Simití y La Luna) (Rolón, 2004), rocas con edad entre el Cretácico Superior y el Paleoceno que corresponden a rocas siliciclásticas marinas a paludales (formaciones de Umir y Lisama) depositadas en ciclos regresivos-transgresivos (Cooper, et al., 1995; Horton, et al., 2010; Rolón, 2004), y, por último, rocas de edad entre el Paleógeno y el Neógeno inicialmente deltaicas, seguidas de rocas continentales depositadas en un ambiente fluvial y rocas clásticas lacustres (formaciones de La Paz, Esmeraldas, Mugrosa, Colorado y Real) (Moreno, et al., 2011; Caballero, et al., 2010; Rolón, 2004).

Modelos del Campo Escuela Colorado. Mediante la interpretación sísmica, Ecopetrol (2003) definió un modelo estructural para el Campo Escuela Colorado controlado por un sistema de fallas inversas paralelas que lo delimita y segmenta en cinco bloques. Por otro lado, con base en la reinterpretación de cuatro líneas sísmicas, Chajín, et al. (2013) propusieron un modelo estructural definido por fallas inversas con anticlinales y retrocabalgamientos asociados (Figura 2). Con base en información de superficie, Zafra (2013) propuso que el anticlinal de Colorado está afectado trasversalmente por fallas normales con componente de rumbo sinestral. a)

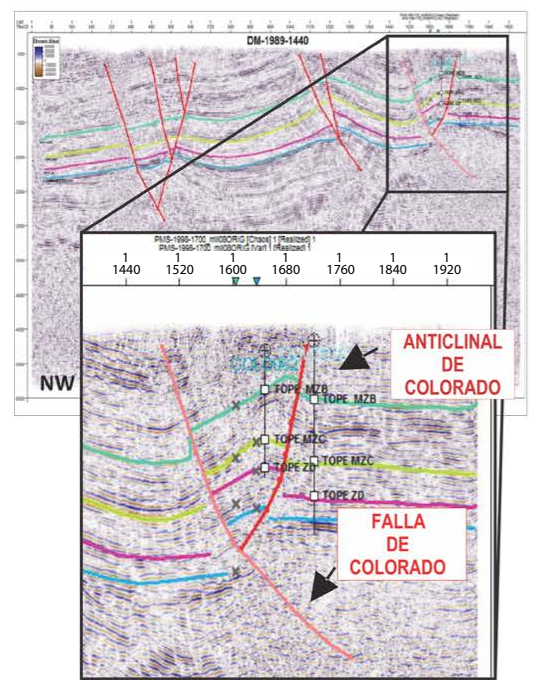

b)

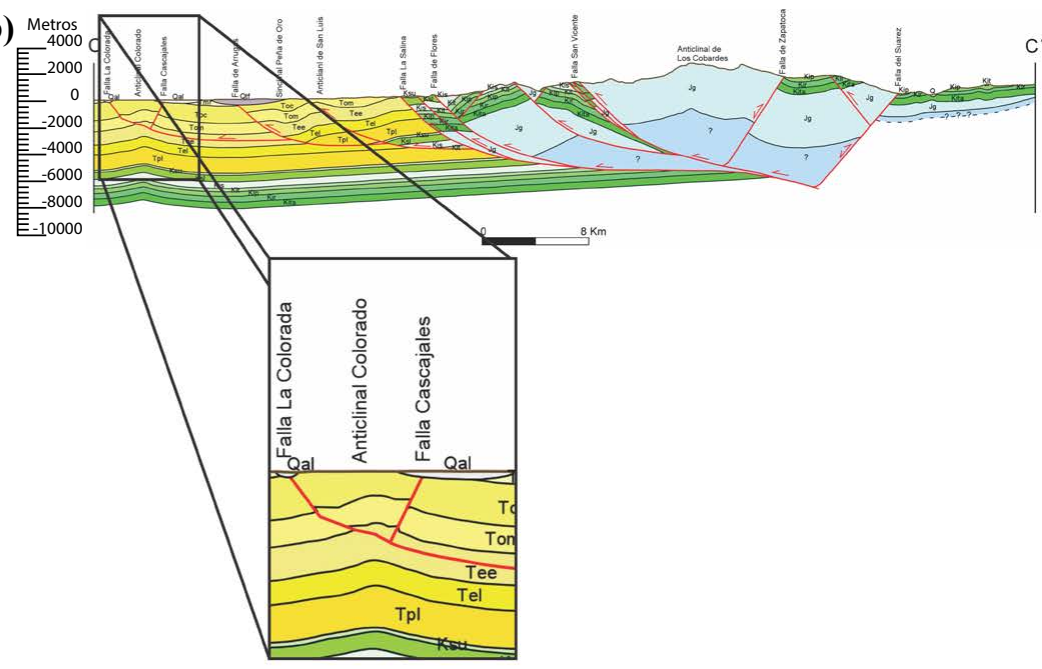

Figura 2. Comparación de: a) la interpretación de líneas sísmicas realizada por Chajín, et al. (2013); b) corte regional realizado por Jiménez, et al. (2016), enfocado en la zona del Campo Escuela Colorado. Gráficas tomadas y modificadas de Chajín, et al. (2013), López \& Jaimes (2015) y Macías \& Cabanzo (2017) 
Condiciones experimentales y escalado. El escalado puede definirse como la relación entre dos cuerpos con igual forma y densidad, pero con dimensiones diferentes. Con una constante de proporcionalidad es posible vincular directamente ambos cuerpos para el análisis de las propiedades físicas en el laboratorio (Hubbert, 1937). Según Graveleau, et al. (2012), en modelos de deformación de la corteza superior se consideran dos comportamientos reológicos: 1) un comportamiento frágil (granitos y secuencias sedimentarias), y 2) un comportamiento viscoso (evaporitas, shales). Estas subdivisiones de la corteza se representan en los modelos análogos con diferentes materiales. Un material no cohesivo, como la arena, con un ángulo de reposo $(\mu)$ de 0,6 a 0,85 es una opción para representar el comportamiento frágil de la corteza superior (Graveleau, et al., 2012; Davis \& Engelder, 1985). Los materiales viscosos, como la silicona, generalmente se emplean para simular la corteza inferior o cuerpos que presenten comportamientos dúctiles (diapiros de lodo o sal) (Dooley \& Schreurs, 2012). El comportamiento frágil se asume como un comportamiento friccional o del tipo Mohr-Coulomb, en el que el escalado entre las propiedades físicas del modelo y la naturaleza ponen en relación la cohesión (C), la densidad $(\rho)$, la gravedad (g) y una dimensión de longitud (L) (Graveleau, et al., 2012).

$$
\mathrm{C}^{*}=\rho^{*} \times \mathrm{L}^{*} \times \mathrm{g}^{*}
$$

El comportamiento viscoso sigue un comportamiento newtoniano, el cual es el parámetro básico del escalado y liga el esfuerzo $(\sigma)$, la viscosidad $(\eta)$ y la tasa de deformación (E) (Graveleau, et al., 2012).

$$
\sigma^{*}=\eta^{*} \times \varepsilon^{*}
$$

En las ecuaciones 1 y 2 la estrella $(*)$ representa el factor de proporcionalidad entre el modelo y la naturaleza para cada parámetro. El factor de tiempo ( $\left.t^{*}\right)$ entre el modelo y la naturaleza puede derivarse de la ecuación 2.

Experimentos, parámetros y materiales. La mesa de deformación utilizada para los experimentos consistió en una base deslizante de $100 \mathrm{~cm}$, con dos paredes de vidrio (Figura 3). Los experimentos se desarrollaron bajo condiciones de gravedad normal. Se utilizó arena cuarzosa, seca, no cohesiva, con un coeficiente de fricción interna de $0,7 \mathrm{y}$ una densidad promedio de $1,58 \mathrm{~g} / \mathrm{cm}^{3}$. Se usó un papel mylar para simular el despegue basal horizontal cuyo coeficiente de fricción es despreciable (McClay, et al., 1999; Davis, et al., 1983). Con base en la ecuación (1) se determinó que el factor de escala era $10^{-5}$, donde $1 \mathrm{~cm}$ en el modelo equivale a $1 \mathrm{~km}$ en la naturaleza.

La configuración experimental fue igual a la utilizada en modelos de cuñas de doble vergencia (Storti, et al., 2000). La deformación es inducida en la arena cuando la película de papel mylar (basamento) se mueve simulando una banda transportadora que genera un prisma de acreción bivergente (Figura 3) al llegar al punto que denominamos barrera cinética, o discontinuidad de velocidad (van der Pluijn \& Marshak, 2004)

Los modelos se construyeron sobre una superficie horizontal y utilizando un tamiz se dispuso la arena capa a capa y con alternancia de colores. En los experimentos el espesor de la arena fue constante, de $3 \mathrm{~cm}$, y la longitud variable, entre 80 y $95 \mathrm{~cm}$ (Figura 3). Las paredes de vidrio se cubrieron con grafito para minimizar la fricción en esta zona del experimento. Al finalizar cada experimento, el modelo se cubrió con arena residual y se procedió a humedecerlo con agua, con el fin de consolidarlo y cortarlo en secciones transversales para observar la geometría interna.

El seguimiento fotográfico del registro transversal y en planta de los experimentos se hizo con dos tipos de cámaras: una Canon EOS T3i y una Go-pro-Silver.

\section{Resultados}

Con el fin de garantizar estadísticamente la reproducibilidad de los experimentos se hicieron 15 pruebas experimentales de cuñas bivergentes con longitudes iniciales (Xo) máximas de $92 \mathrm{~cm}$ y longitudes mínimas de $80 \mathrm{~cm}$ (Tabla 1), y longitudes finales (X) máximas de $78 \mathrm{~cm} *$ y mínimas de $57 \mathrm{~cm}$. Los resultados de los 15 experimentos (Tabla 1, Figura 4) evidenciaron una tendencia en sus estructuras y geometrías repetibles y similares a la de los experimentos reportados en trabajos previos (Malavieille, 1984; Liu, et al., 1992; Biggi, et al., 2010). En el análisis de evolución del modelo, se registraron los datos de longitud (Xo) y altura

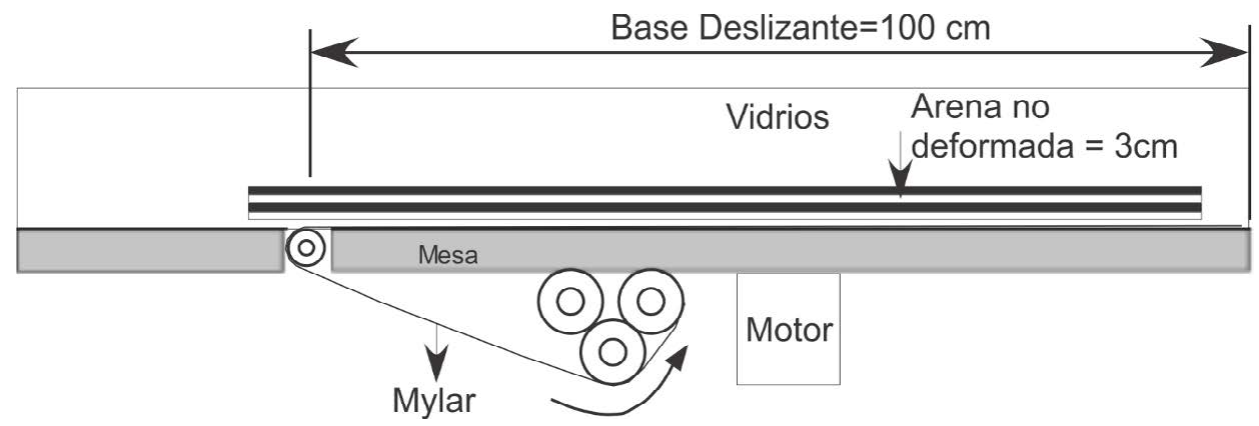

Figura 3. Esquema del montaje inicial de cada uno de los 15 modelos en el que se aprecia la intercalación de capas con un espesor total de $3 \mathrm{~cm}$ y la base deslizante de $100 \mathrm{~cm}$ 
(Yo) iniciales y finales (x, y), con el fin de calcular el porcentaje de acortamiento. En promedio, para los experimentos este fue de $26 \%$ (22 cm) (Tabla 1). Los experimentos mostraron un rápido crecimiento de la zona axial de la cuña debido al apilamiento de material asociado a las estructuras de la retrocuña (Figura 1S, https://www.raccefyn.co/index. php/raccefyn/article/downloadSuppFile/673/2766) (Tabla 1).

En la figura 4 se muestran diferentes cortes trasversales en la prueba $n^{\circ} 15$, en la que se resaltan las fallas generadas en la cuña bivergente. Estas fallas se enumeraron (Figura 4c) según el orden cronológico de crecimiento.

En el análisis de la cuña bivergente establecida en los modelos se puede observar el crecimiento de las fallas de cabalgamiento principales en el frente de la deformación (con vergencia hacia el promontorio) y una serie de retrocabalgamientos en el flanco trasero de la cuña. Las estructuras hacia el frente de la cuña crecieron con dos fallas inversas principales espaciadas cada $10 \mathrm{~cm}$ aproximadamente, definiendo una geometría de cuenca de promontorio (Figura 4). Las fallas de acomodación o retrocabalgamientos en los modelos se asociaron únicamente con la segunda falla principal (Figuras 4 y 5). Esta estructuración se localizó en las zonas más distales de la cuña y se evidenció por la doble vergencia de las fallas que se definen en un despliegue $p o p$ up (Figura 5).

\section{Discusión y conclusiones}

El flanco occidental de la Cordillera Oriental está definido como un frente de deformación netamente inverso o dominado por cizalla pura (Taboada, et al., 2000; Cortés,
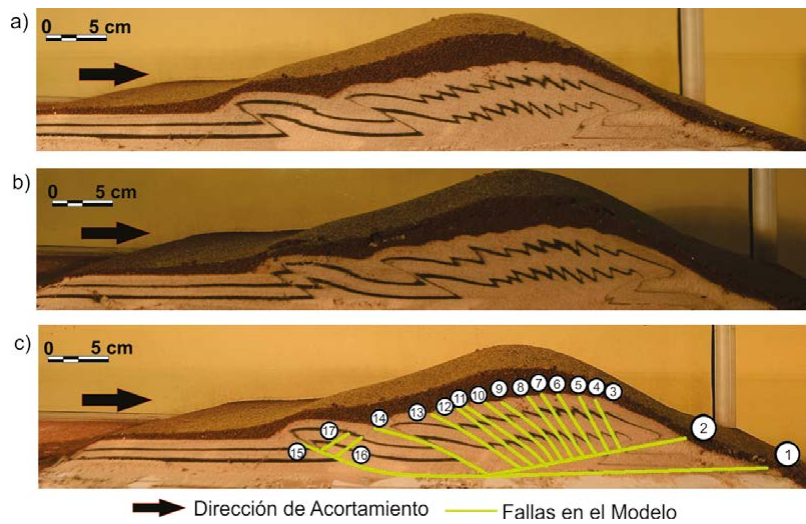

Figura 4. a., b., c. Cortes transversales en el modelo 15 de cuña bivergente. En c. las fallas generadas se enumeraron de 1 a 17 según su orden de crecimiento.

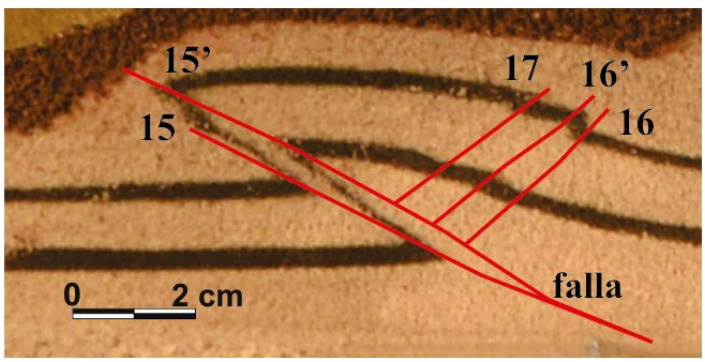

Figura 5. Corte trasversal del modelo 15 en el que se resalta la formación de una falla principal con vergencia al promontorio y cuatro retrocabalgamientos asociados

Tabla 1. Condiciones iniciales (Xo) y finales (X) para el cálculo del porcentaje de elongación (\%e) de los modelos realizados.

\begin{tabular}{|c|c|c|c|c|c|c|c|}
\hline$N^{\circ}$ de serie & $\begin{array}{l}\text { Longitud inicial - Xo } \\
\qquad(\mathrm{cm})\end{array}$ & $\begin{array}{l}\text { Longitud final - X } \\
\text { (cm) }\end{array}$ & $\Delta \mathbf{X}$ & $\begin{array}{l}\text { Altura inicial - Yo } \\
\text { (cm) }\end{array}$ & $\begin{array}{l}\text { Altura final - Y } \\
(\mathbf{c m})\end{array}$ & $\Delta \mathbf{Y}$ & $\%$ e \\
\hline 1 & 80,0 & 57,0 & $-23,0$ & 3 & 9,0 & 6,0 & $-28,8$ \\
\hline 2 & 88,0 & 59,0 & $-29,0$ & 3 & 10,0 & 7,0 & $-33,0$ \\
\hline 3 & 80,0 & 59,0 & $-21,0$ & 3 & 8,5 & 5,5 & $-26,3$ \\
\hline 4 & 80,0 & 57,0 & $-23,0$ & 3 & 8,5 & 5,5 & $-28,8$ \\
\hline 5 & 80,0 & 59,0 & $-21,0$ & 3 & 9,0 & 6,0 & $-26,3$ \\
\hline 6 & 85,0 & 60,0 & $-25,0$ & 3 & 9,0 & 6,0 & $-29,4$ \\
\hline 7 & 85,0 & 61,0 & $-24,0$ & 3 & 9,5 & 6,5 & $-28,2$ \\
\hline 8 & 83,0 & 60,0 & $-23,0$ & 3 & 8,3 & 5,3 & $-27,7$ \\
\hline 9 & 85,0 & 65,0 & $-20,0$ & 3 & 7,7 & 4,7 & $-8,2$ \\
\hline 10 & 85,0 & 78,0 & $-7,0$ & 3 & 8,2 & 5,2 & $-22,2$ \\
\hline 11 & 90,0 & 70,0 & $-20,0$ & 3 & 8,0 & 5,0 & $-32,9$ \\
\hline 12 & 85,0 & 57,0 & $-28,0$ & 3 & 8,0 & 5,0 & $-32,9$ \\
\hline 13 & 88,0 & 64,0 & $-24,0$ & 3 & 8,3 & 5,3 & $-27,3$ \\
\hline 14 & 92,0 & 65,0 & $-27,0$ & 3 & 8,8 & 5,8 & $-29,3$ \\
\hline 15 & 80,0 & 57,0 & $-23,0$ & 3 & 9,5 & 6,5 & $-28,8$ \\
\hline Promedio & 84,4 & 61,9 & $-22,5$ & 3 & 8,7 & 5,7 & $-26,7$ \\
\hline
\end{tabular}


et al., 2006). En las series experimentales realizadas en este trabajo se consideró una deformación coaxial o de cizalla pura, y durante los experimentos no se consideraron factores como las anisotropías de basamento, las fallas transversales o la cizalla simple. Para los 15 experimentos se asumió esta condición como condición límite, por lo tanto, los modelos representan una cuña bivergente caracterizada por fallas netamente de cabalgamiento con vergencia al promontorio y con pliegues asociados (Figura 5 y 6 ).
Con base en las características estructurales definidas en los 15 modelos experimentales es posible definir el Campo Escuela Colorado dentro de un estilo estructural de piel delgada, caracterizado por fallas inversas con vergencia hacia el oeste y desarrollo de pliegues por propagación de falla y retrocabalgamientos en las zonas más distales del frente de deformación de la Cordillera Oriental (Figura 6 y 7). Los resultados son coherentes con el marco estructural del sector sureste del Valle Medio del Magdalena, el cual

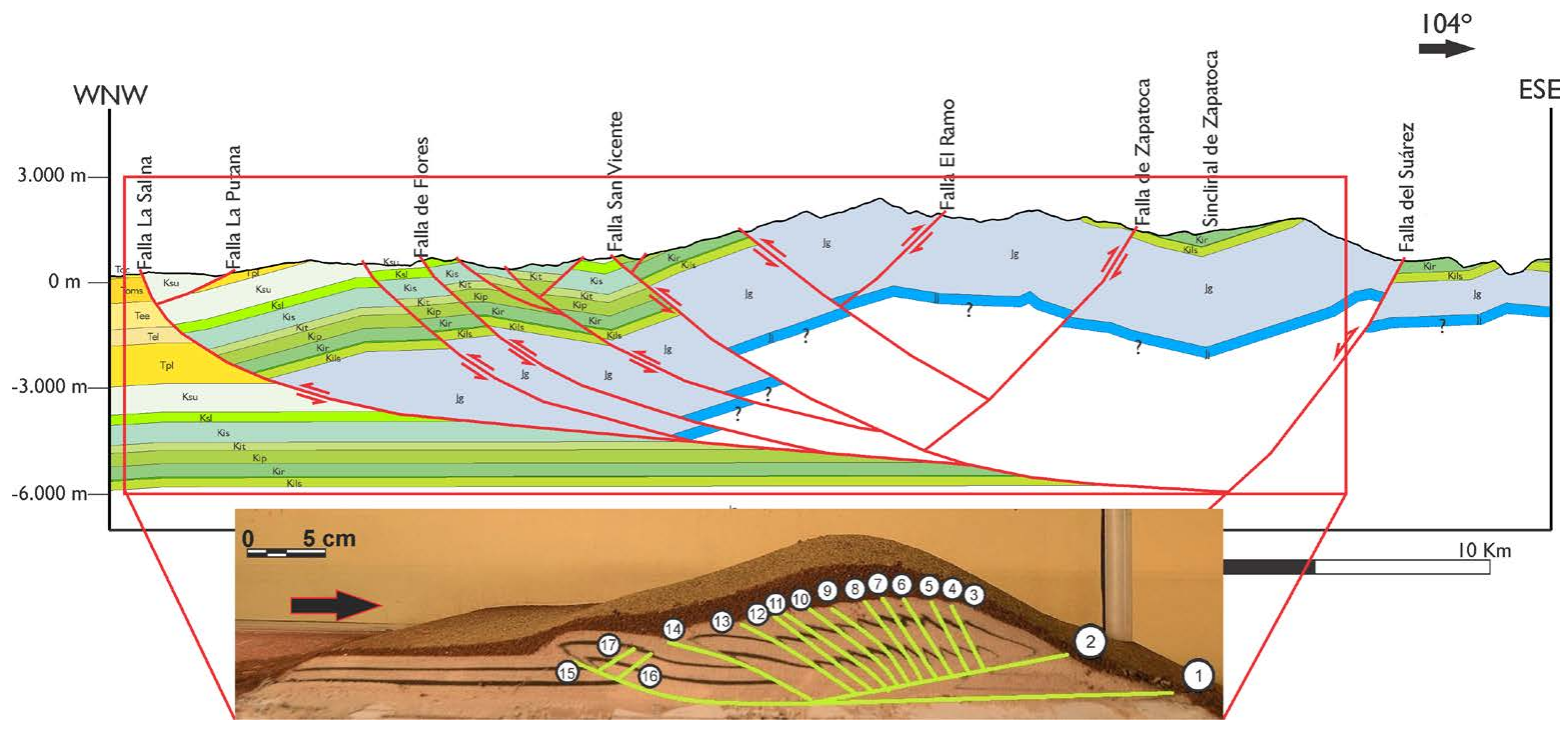

Figura 6. Corte regional de la cuenca del Valle Medio del Magdalena (Macías \& Cabanzo, 2017) y corte transversal del modelo de cuña bivergente. En los dos esquemas se resaltan fallas inversas principales con el desarrollo de retrocabalgamientos y pliegues asociados.

a)

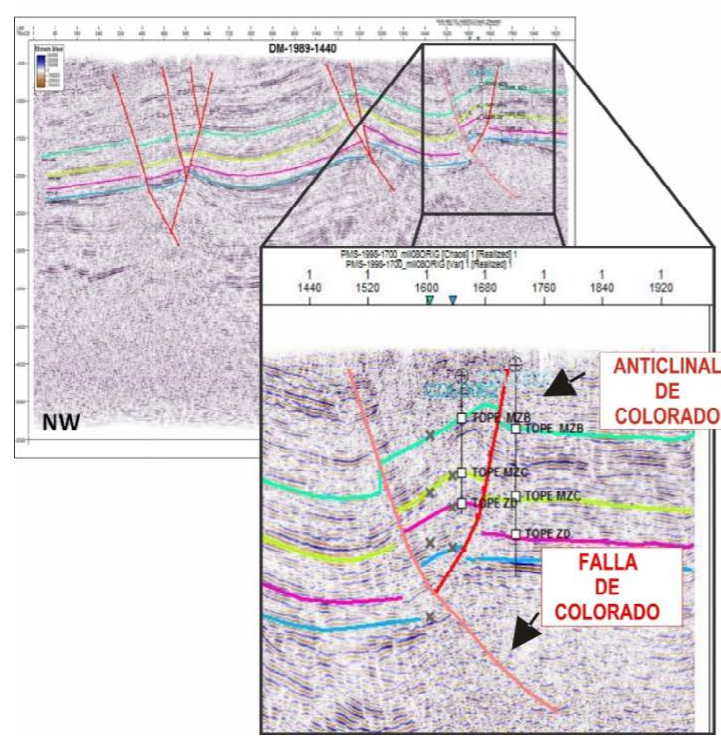

b)

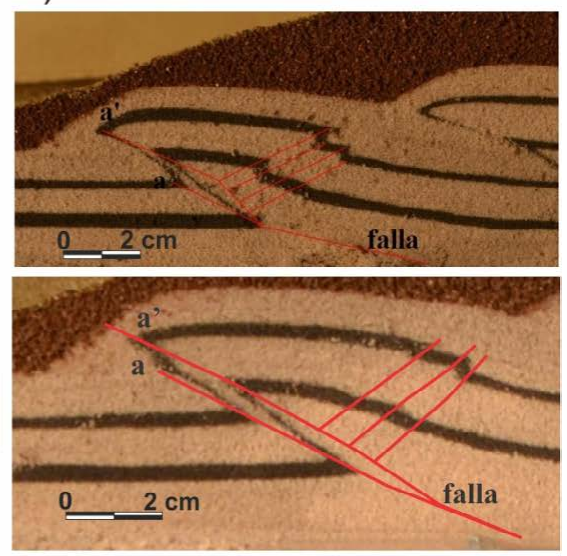

Figura 7. Comparación entre: a) el modelo estructural del Campo Escuela Colorado de Chajín, et al. (2013) y b) el resultado de los experimentos realizados. a) Modelo estructural definido por la falla de Colorado con vergencia inversa hacia el oeste y su anticlinal y retrocabalgamiento asociados. Interpretación de la línea sísmica DM-1989-1440. b) Corte transversal del modelo a escala de laboratorio en el que se muestra una falla principal con vergencia al promontorio y retrocabalgamientos asociados 
según Córdoba, et al. (2001), se define como una cadena plegada y fallada con vergencia hacia el oeste (fallas de Bituima y La Salina) e intervalos de despegue en las formaciones de La Luna y Umir.

La cuña sedimentaria en los experimentos se caracterizó por acortamientos constantes en el rango de 20 a $30 \%$, con ligeras variaciones entre los modelos con la misma longitud inicial. Según Pierson (2014), las variaciones de acortamiento pueden explicarse por el desplazamiento de las fallas y por la acomodación granular debida al acortamiento paralelo a las capas. La zona axial de la cuña se caracterizó por elevaciones topográficas rápidas en las primeras fases de deformación, con un apilamiento de material y engrosamiento hasta duplicar el espesor de la cobertera (Figura 1S, https://www.raccefyn.co/index.php/raccefyn/article/download SuppFile/673/2766 y Tabla 1).

Según los resultados obtenidos en los 15 experimentos realizados y con las condiciones límites definidas, no se encontraron estructuras de alto ángulo, independientes o que segmentaran la cuña. Además, no se observaron estructuras con componente de rumbo o deformación no coaxial, por lo que los resultados difieren de las interpretaciones realizadas en los modelos de Ecopetrol (2003) y Zafra (2013). Asimismo, el estilo estructural definido en los experimentos y los resultados observados (Figura 6) concuerdan y presentan similitudes con el corte regional realizado por Garavito (200)8, Araque \& Otero (2016), Jiménez, et al. (2016) y Macías \& Cabanzo (2017). Teniendo en cuenta el estilo estructural de piel delgada y la geometría de las estructuras definidas por un frente de deformación con retrocabalgamientos, se concluye que los resultados concuerdan con el modelo estructural propuesto por Chajín, et al. (2013) para el Campo Escuela Colorado.

\section{Información suplementaria}

Figura 1S. Secuencia completa de la evolución de la cuña bivergente del experimento $\mathrm{n}^{\circ} 15$ : a) etapa inicial y q) etapa final. Esta imagen evidencia el crecimiento de la estructura asociado a las fallas de la retrocuña enumeradas según su etapa de crecimiento. Vea la figura 1S en: https://www.raccefyn.co/index.php/raccefyn/ article/downloadSuppFile/673/2766

\section{Agradecimientos}

Agradecemos a la administración del Campo Escuela Colorado por colaborarnos con la información requerida para esta publicación. Además, agradecemos a los revisores anónimos y al editor ya que sus comentarios y sugerencias permitieron mejorar el manuscrito.

\section{Contribución de los autores}

El presente artículo, fue elaborado y revisado por Camilo Anaya, Giovanny Jiménez Díaz y Dilan A. Martínez Sánchez. Camilo Anaya realizó los experimentos bajo la supervisión de Giovanny Jimenez. Las figuras y tablas fueron diseñadas, realizadas y discutidas por los tres autores. La redacción del texto fue liderada por Camilo Anaya y Dilan A. Martinez, Giovanny Jimenez participó en la redacción de la introducción y la discusión. El documento final es un aporte equitativo de los tres autores.

\section{Conflicto de intereses}

Ninguno

\section{Referencias}

Araque-G., C. N. \& Otero-R., J. L. (2016). Zonas transversales y su relación con estructuras regionales, flanco O-Cordillera Oriental. Tesis de Grado, Universidad Industrial de Santander.

Barrero, D., Pardo, A., Vargas, C., Martínez, J. (2007). Colombian sedimentary basins: Nomenclature, boundaries and petroleum geology, a new proposal. Agencia Nacional de Hidrocarburos - ANH. Editado y compilado por ANH and B\&M Exploration Ltda. Bogotá, Colombia.

Bayona, G., Cortés, M., Jaramillo, C., Ojeda, G., Aristizabal, J. J., Reyes-Harker, A. (2008). An integrated analysis of an orogen-sedimentary basin pair: Latest CretaceousCenozoic evolution of the linked Eastern Cordillera orogen and the Llanos foreland basin of Colombia. Bulletin of the Geological Society of America, 120 (9-10): 1171-1197. doi: 10.1130/B26187.1

Bigi, S., Di Paolo, L., Vadacca, L., Gambardella, G. (2010). Load and unload as interference factors on cyclical behavior and kinematics of Coulomb wedges: Insights from sandbox experiments, Journal of Structural Geology. 32: 28-44.

Brun, J. P. (2002). Deformation of the continental lithosphere: Insights from brittle-ductile models. En: Deformation Mechanisms, Rheology and Tectonics: Current Status and Future Perspectives. Editores: S. De Meer, et al., Geol. Soc. Spec. Publ. 200: 355-370.

Caballero, V., Parra, M., Mora, A. (2010). Levantamiento de la Cordillera Oriental de Colombia durante el Eoceno tardío - Oligoceno temprano: proveniencia sedimentaria en el sinclinal de Nuevo Mundo, cuenca Valle Medio del Magdalena. Boletín de Geología. 32 (1): 45-77.

Chajín, P., Mier, R., Palmera, T. Delgado, L., Luna, J. (2013). Reinterpretación del modelo estructural del Campo Escuela Colorado, VMM, Colombia. En XV Congreso Colombiano de Petrólero y Gas. Noviembre 20 al 22. Corferias, Bogotá.

Cooper, M. A., Addison, F. T., Álvarez, R., Coral, M., Graham, R. H., Hayward, S. H., Martínez, J., Naar, J., Peñas, R., Pulham, A. J., Taborda, A. (1995). Basin development and tectonic history of the Llanos Basin, Eastern Cordillera, and Middle Magdalena Valley, Colombia. American Association of Petroleum Geologists Bulletin. 79 (10): 1421-1443.

Córdoba, F., Rolón, L., Buchelli, F., Suárez, M. (2000). Provincia petrolífera del Valle Medio del Magdalena, Colombia. Empresa Colombiana de Petróleos (ECOPETROL). $7^{\circ}$ Simposio Bolivariano - Exploración Petrolera en las Cuencas Subandinas. Caracas, Venezuela. p. 603-605.

Córdoba, F., López, C., Rolón, L., Buchelli, F., Sotelo, C. (2001). Proyecto evaluación regional del Valle Medio del Magdalena y Cordillera, Ecopetrol, informe interno: Bogotá. 
Cortés, M., Colletta, B., Angelier, J. (2006), Structure and tectonics of the central segment of the Eastern Cordillera of Colombia, J. S. Am. Earth Sci. 21: 437-465, doi:10.1016/j. jsames.2006.07.004

Cristallini, E. (1998). Introducción a las fajas plegadas y corridas. Buenos Aires, Argentina.

Davis, D., Suppe, J., Dahlen, F.A. (1983). Mechanics of fold and thrust belts and accretionary wedges. Journal of Geophysical Research. 88: 1153-1172.

Dooley, T. P. \& Schreurs, G. (2012). Analogue modelling of intraplate strike-slip tectonics: A review and new experimental results. Tectonophysics. 574: 1-71.

Ecopetrol. (2003). Diagnóstico y estrategias de recobro Campo Colorado, Gerencia Magdalena Medio, Instituto Colombiano de Petróleo. Bogotá. p. 126.

Etayo-Serna, F. (1968). El sistema Cretáceo en la región de Villa de Leiva y zonas próximas. Geología Colombiana. 5: 5-74.

Fabre, A. (1983). La subsidencia de la Cuenca del Cocuy (Cordillera Oriental de Colombia) durante el Cretáceo y el Terciario Inferior. Primera parte: Estudio cuantitativo de la subsidencia. Geología Norandina. 8: 22-27.

Garavito, C. F. (2008). Structural analysis of an area in the northern central part of the Middle Magdalena Valley Basin, Colombia. Tesis de Maestría, Colorado School of Mines.

Graveleau. F., Malavieille, J., Dominguez, S. (2012). Experimental modelling of orogenic wedges: A review. Tectonophysics. 538-540: 1-66

Horton, B.K., Saylor, J.E., Nie, J., Mora, A., Parra, M., ReyesHarker, A., Stockli, D.F. (2010). Linking sedimentation in the northern Andes to basement configuration, Mesozoic extension, and Cenozoic shortening: Evidence from detrital zircon U-Pb ages, Eastern Cordillera, Colombia. GSA Bulletin. 12: 1423-1442.

Hubbert, M. K. (1937). Theory of scale models as applied to the study of geologic structures. Geological Society of America Bulletin. 48 (10): 1459-1520.

Jiménez, G., López, O., Jaimes, L., Mier U., R. (2016). Variaciones en el estilo estructural relacionado con anisotropías de basamento en el Valle Medio del Magdalena. Revistas Académicas Ciencias de la Tierra. 40 (155): 312-319.

Liu, H., McClay, K.R., Powell, D. (1992). Physical models of thrust wedges. En: McClay, K.R. (Editores). Thrust Tectonics. New York, USA: Chapman and Hall. p. 71-81.

López. O. A. \& Jaimes. E. L. (2015). Contribución al conocimiento geológico del sector Sureste de la Plancha 120-II-C, Valle Medio del Magdalena. Tesis de Grado, Universidad Industrial de Santander, Bucaramanga, Colombia.

Macías, Y. \& Cabanzo, M. (2017). Análisis y caracterización estructural de zonas transversales en el bloque colgante de la falla del Suárez (Tesis de pregrado). Universidad Industrial de Santander, Bucaramanga, Colombia.

McClay, K., Dooley, T., Whitehouse, P. (1999). Analogue modeling of thin and thick-skinned thrust systems. Thrust Tectonics. Egham, U.K. p. 26-29.

Malavieille, J. (1984). Modelisation experimentale des chevauchements imbriques: Application aux chaines de montagnes. Bulletin de la Societe' Geologique de France. 7: 129-138.
Mojica, J. \& Franco, R. (1990): Estructura y Evoluci6n Tectónica del Valle Medio y Superior del Magdalena. Geología Colombiana. 17 (14): 41-64.

Moreno, C. J., Horton, B. K., Caballero, V., Mora, A., M., Sierra, J. (2011). Depositional and provenance record of the Paleogene transition from foreland to hinterland basin evolution during Andean orogenesis, northern Middle Magdalena Valley Basin, Colombia. Journal of South American Earth Sciences. 32: 246-263.

Nie, J., Horton, B. K., Saylor, J. E., Mora, A., Mange, M., Garzione, C. N., ... Parra, M. (2012). Integrated provenance analysis of a convergent retroarc foreland system: U-Pb ages, heavy minerals, $\mathrm{Nd}$ isotopes, and sandstone compositions of the Middle Magdalena Valley basin, northern Andes, Colombia. Earth-Science Reviews. 110 (1-4): 111-126. doi: 10.1016/j.earscirev.2011.11.002

Pierson, N. (2014). Assessing Layer Parallel Shortening in the Eastern Colorado Front Range Using Thin Section Analysis and Analog Sandbox Models (Thesis of Master of Science). University of Nebraska, Lincoln.

Portillo, R. (2008). Influencia de la heterogeneidad mecánica de medios estratificados en el estilo y evolución estructural durante acortamiento: experimentos físicos. (Tesis de pregrado). Instituto Politécnico Nacional, Mexico DF, méxico.

Restrepo-Pace, P. A., Colmenares, F., Higuera, C., Mayorga, M. (2004). A fold and thrust belt along the western flank of the Eastern Cordillera of Colombia-Style, kinematics, and timing constraints derived from seismic data and detailed surface mapping. En K. R. McClay (Editores). Thrust tectonics and hydrocarbon systems: AAPG Memoir. 82: 598-613.

Rolón, L. F. (2004). Structural Geometry of the Jura-Cretaceous Rift of the Middle Magdalena Valley Basin-Colombia. Department of Geology and Geography. Thesis of Master of Science in Geology, West Virginia University. Morgantown, WV, USA.

Sarmiento-Rojas, L. F., van Wess, J. D., Cloetingh, S. (2006), Mesozoic transtensional basin history of the Eastern Cordillera, Colombian Andes: Inferences from tectonic models, J. S. Am. Earth Sci., 21: 383-411. doi:10.1016/j. jsames.2006.07.003

Storti, F., Salvini, F., McClay, K. (2000). Synchronous and velocity-partitioned thrusting and thrust polarity reversal in experimentally produced, doubly-vergent thrust wedges: Implications for natural orogens. Tectonics. 19 (2): 378-396.

Taboada, A., Rivera, L. A., Fuenzalida, A., Cisternas, A., Philip, H., Bijwaard, H., Olaya, J., Rivera, C. (2000). Geodynamics of the northern Andes: Subductions and intracontinental deformation (Colombia). Tectonics. 19: 787-813.

Taborda, B. (1965). Guide book to the Geology of the De Mares Concession. Geotec, Bogotá

van Der Pluijm, B. A. \& Marshak, S. (2002). Earth Structure: An introduction to structural geology and tectonics. Norton \& Company inc. New York.

Zafra, E. (2013). Cartografía geológica del Campo Colorado a escala 1: 25.000. Tesis de Pregrado, Universidad Industrial de Santander, Bucaramanga, Colombia. 